For by Proposition 4, $F A=A$ for every $A$ and $A\left(A^{-1} F\right)=E F=F$.

Let $S_{1}, \cdots, S_{n}$ be $n$ statements. Let $A_{i}$ be the statement: "All the preceding statements are annulled but $S_{i}$ is true." It is interesting to note that the statements $A_{i}$ form an idempotent $(l, r)$ system.

Ohio State University

\title{
A CONJECTURE IN ELEMENTARY NUMBER THEORY
}

\section{LEON ALAOGLU AND PAUL ERDÖS}

A well known conjecture of Catalan states that if $f(n)$ is the sum of all divisors of $n$ except $n$, then the sequence of iterates of $f(n)$ is either eventually periodic or ends at 1 . It not only seems impossible to prove this, but it is also very difficult to verify. ${ }^{1}$

Another conjecture of Poulet, ${ }^{2}$ which appears equally difficult to prove, has the doubtful merit that it is easy to verify. Let $\sigma(n)$ be the sum of all divisors of $n$, and let $\phi(n)$ be Euler's function. Then for any integer $n$ the sequence

$$
f_{0}(n)=n, \quad f_{2 k+1}(n)=\sigma\left(f_{2 k}(n)\right), \quad f_{2 k}(n)=\phi\left(f_{2 k-1}(n)\right)
$$

is eventually periodic.

We have verified this conjecture to $n=10000$ (extending Poulet's verification) by using Glaisher's tables. ${ }^{3}$ The checking was facilitated by the following observation: if the conjecture is to be checked for all $n<x$, it is enough to find a member of the sequence other than the first which is less than $x$.

The longest cycle found was in the sequence $f_{k}(9216)$. It starts with $f_{6}(9216)$, and is: $34560,122640,27648,81800,30976,67963,54432$, $183456,48384,163520,55296,163800,34560$. However our method of checking does not show that this is the largest cycle up to 10000 , and in fact Poulet found that $f_{k}(1800)$ has the same length 12.

As a rule $\phi(\sigma(n))$ is less than $n$. In fact, it can be shown that for every $\epsilon>0, \phi(\sigma(n))<\epsilon n$, except for a set of density 0 . The proof follows from the following two observations:

Received by the editors May 18, 1944.

${ }^{1}$ L. E. Dickson, Theorems and tables on the sum of the divisors of a number, Quart. J. Math. vol. 44 (1913) pp. 264-296, and P. Poulet, La Chasse aux Nombres, vol. 1, pp. 68-72, and vol. 2, p. 188.

2 P. Poulet, Nouvelles suites arithmetiques, Sphinx vol. 2 (1932) pp. 53-54.

${ }^{3} \mathrm{~J}$. W. L. Glaisher, Number-divisor tables, British Association for the Advancement of Science, Mathematical Tables, vol. 8. 
(1) For a given prime $p$, the set of all $n$ such that $\sigma(n) \equiv 0(\bmod p)$ is of density 1.

The set of all integers not divisible by any prime $q$ of the form $p x-1$ is of density zero, since $\sum_{q} 1 / q$ diverges. Hence the set of all integers divisible by a prime $q>N$ of this type is of density 1 . But the set of all integers divisible by $q^{2}, q>N$, is of density less than $\sum_{q>N} 1 / q^{2}=o(1)$. Therefore, if $x$ is large, the number of $n$ less than $x$ such that $\sigma(n) \equiv 0(\bmod p)$ exceeds $(1-\epsilon) x$.

(2) Except for $\epsilon x$ integers $n$ less than $x, \sigma(n)<c(\epsilon) n$.

This follows from the fact that $\sum_{n<x} \sigma(n) \sim \pi^{2} n^{2} / 12$.

Choose $p$ so that $\prod_{q \leqq p}(1-1 / q)<\delta / c(\epsilon)$. Then, if $x$ is sufficiently large, all except $\eta x+\epsilon x$ integers $n$ less than $x$ have $\sigma(n)<c(\epsilon) n$, $\sigma(n) \equiv 0(\bmod q)$ for all $q \leqq p$. But, with these exceptions, $\phi[\sigma(n)]<\delta n$, which completes the proof, since $\eta$ and $\epsilon$ are arbitrary.

In much the same way it can be shown that for every $c>0, \sigma[\phi(n)]$ $>$ cn except for a set of density zero.

Actually, much more can be shown. Except for a set of density zero, $e^{\gamma} \phi[\sigma(n)] \log \log \log n \sim \sigma(n)$, and $e^{-\gamma} \sigma[\phi(n)] / \log \log \log n \sim \phi(n)$, where $\gamma$ is Euler's constant. The proof is suppressed, but it might be noted that the reason for this result is that, for almost all $n, \phi(n)$ and $\sigma(n)$ are both divisible by all primes less than $(\log \log n)^{1-\epsilon}$, and by relatively few primes greater than $(\log \log n)^{1+\epsilon}$.

There exist numbers for which $\phi(\sigma(n))=n$. Up to 2500 these numbers are $1,2,8,12,128,240,720$; while two further solutions are $2^{15}$ and $2^{31}$. Poulet gives many others; we do not know whether there are infinitely many solutions.

We state two further conjectures:

(a) Form the sequence $\sigma(n), \sigma(\sigma(n)), \phi(\sigma(\sigma(n))), \sigma(\phi(\sigma(\sigma(n))))$ in which the functions are successively applied in the order $\sigma, \sigma, \phi$, $\sigma, \sigma, \phi, \sigma, \sigma, \phi, \ldots$. This sequence seems to tend to infinity if $n$ is large enough.

(b) On the other hand, the sequence $\phi(n), \phi(\phi(n)), \sigma(\phi(\phi(n))), \cdots$, in which the order is $\phi, \phi, \sigma, \phi, \phi, \sigma, \phi, \phi, \sigma, \cdots$, seems to converge to 1 , for all $n$.

Obviously many more such conjectures can be formulated.

Purdue University 